\title{
Interplay between thin silver films and epitaxial graphene
}

Ivan Shtepliuk, Ivan Gueorguiev Ivanov, Nikolaos Pliatsikas, N. Ben Sedrine, O. Andersson, Tihomir Iakimov, Andreas Jamnig, Kostas Sarakinos and Rositsa Yakimova

The self-archived postprint version of this journal article is available at Linköping University Institutional Repository (DiVA):

http://urn.kb.se/resolve?urn=urn:nbn:se:liu:diva-163667

N.B.: When citing this work, cite the original publication.

Shtepliuk, I., Ivanov, I. G., Pliatsikas, N., Ben Sedrine, N., Andersson, O., Iakimov, T., Jamnig, A., Sarakinos, K., Yakimova, R., (2020), Interplay between thin silver films and epitaxial graphene, Surface \& Coatings Technology, 381, 125200. https://doi.org/10.1016/j.surfcoat.2019.125200

Original publication available at:

https://doi.org/10.1016/j.surfcoat.2019.125200

Copyright: Elsevier

http://www.elsevier.com/

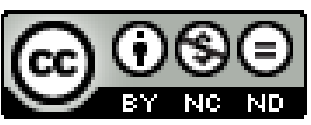




\title{
Interplay between thin silver films and epitaxial graphene
}

\author{
I. Shtepliuk ${ }^{1,2 *}$, I. G. Ivanov ${ }^{1}$, N. Pliatsikas ${ }^{1}$, N. Ben Sedrine ${ }^{3}$, O. Andersson ${ }^{1}$, T. Iakimov ${ }^{1}$, \\ A. Jamnig ${ }^{1}$, K. Sarakinos ${ }^{1}$, and R. Yakimova ${ }^{1}$ \\ ${ }^{1}$ Department of Physics, Chemistry and Biology, Linköping University, SE-58183, \\ Linköping, Sweden \\ ${ }^{2}$ Frantsevich Institute for Problems of Materials Science, NASU-National Academy of \\ Sciences of Ukraine, 142 Kyiv, Ukraine \\ ${ }^{3}$ Departamento de Física \& I3N, Universidade de Aveiro, Campus Universitário de Santiago, \\ 3810-193 Aveiro, Portugal
}

\begin{abstract}
Thin Ag films, with nominal thickness in the range 2 to $30 \mathrm{~nm}$, are deposited using direct current magnetron sputtering and film morphology is studied by means of plan-view scanning electron microscopy. We find that for $2 \mathrm{~nm}$ nominal thickness the film surface consists of isolated circular nanoscale islands, which become interconnected as further material is deposited, leading to a continuous film at a nominal thickness of $30 \mathrm{~nm}$. Our experimental findings are discussed in the context of the density functional theory results, which show that van der Waals forces dominate the interaction between Ag and epitaxial graphene. We also performed microRaman analysis and we find that the $G$ and $2 D$ modes of epitaxial graphene exhibit a red-shift upon Ag-layer deposition; which is interpreted as a result of charge transfer at the Ag/graphene interface. Moreover, we observed a pronounced enhancement of the $G$ peak amplitude and area irrespective of the film nominal thickness and morphology, which we attribute to a combination of the charge transfer and plasmonic resonance effects. Our observations provide a critical information on the interaction between $\mathrm{Ag}$ and epitaxial graphene, which can be useful to design electronic and sensing devices based on Ag-epitaxial graphene hybrids.
\end{abstract}

Keywords: epitaxial graphene, SiC, magnetron sputtering, silver, Raman

*Corresponding author: ivan.shtepliuk@liu.se

\section{Introduction}

Two-dimensional materials (2DMs) are recognized as promising components of nextgeneration miniaturized devices, and they are expected to play a great role in the development of smart and biocompatible applications in medicine, sustainable electronics, and environmental sensorics [1]. Nowadays, 2DMs research activities are at the forefront of scientific attainments $[2,3]$, promoting a discovery of unusual physical phenomena and offering 
avenues of novel technical concepts $[4,5]$. A special focus is placed on the investigation of graphene as a main star of the 2DMs family [6], which exhibits the potential to become a multifunctional platform for on-demand design of different devices. In this regard, an integration of graphene with traditional semiconducting materials (for instance, $\mathrm{SiC}$ ) and noble metals (especially with $\mathrm{Ag}$ as a most prominent representative) may not only complement graphene for advanced technologies, but also allow designing of new hybrid materials with extraordinary performance in catalysis, sensing, optics and electronics. The choice of Ag as a representative noble metal can be justified by following main reasons: (i) silver has outstanding electrical and thermal properties (the highest electrical/thermal conductivity among all metals and the lowest contact resistance of any metal [7]) providing excellent prerequisites for the exploitation of silver as electrical contacts and conductors, and (ii) silver is recognized as the best low-loss plasmonic material, which is an essential condition for development of Localized Surface Plasmon Resonance (LSPR) applications in the visible and near-infrared (NIR) ranges [8].

Due to the interesting combination of intrinsic properties of $\mathrm{Ag}$, graphene, and $\mathrm{SiC}$ (plasmonic nature of $\mathrm{Ag}[9,10]$, ballistic transport in graphene [11, 12] and high breakdown voltage of $\mathrm{SiC}$ [13]), such hybrid systems can retain the advantages of individual constituents and enable to design high-performance devices. For instance, Ag/graphene/SiC hybrid systems can be used in optical sensing and Raman imaging, in which the analysis of molecules and biomaterials is facilitated by surface enhanced Raman scattering (SERS) provided by plasmonic silver nanoparticles [14-17]. Furthermore, over the last few years, there has been an upsurge of interest in the synthesis of two-dimensional metallic films due their potential to be used as key components in gas sensing and catalysis [18]. In this framework, density functional theory (DFT) calculations have suggested that graphene can be an excellent template for growth of stable 2D metallic films [18, 19].

A closer look into the electronic properties of silver [20,21] and epitaxial graphene [22, 23] enables capitalizing another possible benefit originating from the hybridization of the two materials. Silver nanoparticles residing on free-standing graphene are responsible for $n$-type doping of initially neutral graphene [24], which is associated with the work function difference between the two materials. It is also known that epitaxial graphene on $\mathrm{SiC}$ is naturally $n$-doped material due to the charge transfer phenomena at the reconstructed interface [25], and thus there is a possibility of reaching high doping level via fine tuning of the Ag-layer morphology. Abovementioned aspects are essential prerequisites for achieving large-area uniform $n$-type doped epitaxial graphene, while maintaining its quality, and thereby fabricate electronic devices 
( $p$ - $n$ junctions, $p-n-p$ and $n-p-n$ transistors, Schottky diodes) exhibiting reproducible performance.

The graphene structure largely governs the Ag-layer nucleation and growth mechanisms $[16,26]$. Hence, a fundamental understanding of the interplay between Ag and the underlying graphene substrates is required for growing $\mathrm{Ag}$ films with controlled morphology and microstructure. There have been many studies of the interactions between Ag layers and freestanding (i.e., exfoliated) graphene [27-32], but a detailed understanding of the complex interplay among the various layers in the $\mathrm{Ag} / \mathrm{Graphene} / \mathrm{SiC}$ system has not been reached yet. Growth of $\mathrm{Ag}$ on graphene/6H-SiC has been studied by thermal evaporation [15], Ag deposition from chemical solution [14], and evaporation from a water-cooled Knudsen cell at a substrate temperature of $77 \mathrm{~K}[16,26]$. Direct evidences suggest at the initial growth stages isolated $\mathrm{Ag}$ islands exhibit van-der-Waals epitaxy on defect-free monolayer graphene (with measured distance between $\mathrm{Ag}$ and graphene of approximately $3.14 \AA$ ) [15] and substitutional doping of Ag or even Ag intercalation beneath topmost graphene layer take place [26]. It was argued that Ag island size is slightly dependent on epitaxial graphene thickness and the thickness increase causes a reduction of $n$-type doping due to the weakened interaction between $\mathrm{Ag}$ islands and graphene layers $[16,26]$. To obtain deeper understanding of the way by which Ag interacts with epitaxial graphene, Homa et al. [33] investigated the wettability between liquid Ag and graphene-coated $\mathrm{SiC}$ by dispensed drop method at $970^{\circ} \mathrm{C}$ and concluded that a strong chemical interaction at the interface results in the formation of a second graphene layer on top of $\mathrm{Ag}$, local dissolution of $\mathrm{C}$ into liquid $\mathrm{Ag}$, and appearance of local discontinuities in epitaxial graphene. Furthermore, it has been recently shown that ultra-high vacuum annealing of $\mathrm{Ag}$ at $570 \mathrm{~K}$ causes a pronounced sliding of $\mathrm{Ag}$ islands on epitaxial graphene grown on $6 \mathrm{H}-\mathrm{SiC}$ [34]. As a result of such sliding, highly orientated micro-trenches on epitaxial graphene were formed, thereby allowing graphene patterning.

Despite the knowledge generated so far, it is still not understood what the nature of graphene defects induced by Ag deposition is and how Ag deposits of various thicknesses and morphologies influence the quality and vibrational properties of epitaxial graphene. In the present work, we contribute to this understanding by studying the growth of $\mathrm{Ag}$ on graphene/4H-SiC by direct magnetron sputtering.

\section{Experimental procedures}

Monolayer (ML) epitaxial graphene with high thickness uniformity was synthesized by high-temperature thermal decomposition of $\mathrm{Si}$-face $4 \mathrm{H}-\mathrm{SiC}$ substrates (nominally on-axis) in argon atmosphere [35]. Due to the unintentional misorientation of the $\mathrm{SiC}$ substrate and a 
natural step bunching, epitaxial graphene on the $\mathrm{SiC}$ template has terrace-stepped morphology. According to reflectance mapping (not shown here) [36], the SiC surface is up to $97 \%$ covered by monolayer graphene. Bilayer patches are rarely observed. Thus, Ag layer deposition is expected almost entirely on a monolayer graphene.

Thin Ag films were deposited on as-grown epitaxial graphene substrates by direct current (dc) magnetron sputtering in an ultra-high vacuum chamber (based pressure $\sim 10^{-8} \mathrm{~Pa}$ ) at room temperature. Ar gas (purity 99.999\%) at pressures of 0.84 and $1.7 \mathrm{~Pa}$ was used to generate plasma and sputter a magnetron source which was equipped with Ag target (diameter $76.2 \mathrm{~mm}$; thickness $6 \mathrm{~mm}$; purity 99.99\%). The nominal thickness of the Ag films was varied between 2$30 \mathrm{~nm}$ by changing the deposition time. The thicknesses of the different Ag layers correspond to nominal thicknesses $\left(d_{\mathrm{Ag}}\right)$, which were calculated by using following equations:

$$
\left\{\begin{array}{c}
d_{A g}=F \times t \\
F(1 \AA / s)=\frac{d_{c l}}{t}
\end{array}\right.
$$

where $F$ is the deposition rate, $t$ is the deposition time, and $d_{c l}$ is the thickness of a continuous Ag layer, which was determined using spectroscopic ellipsometry in accordance to methodology presented by Elofsson et al. [37].

The film crystal structure was investigated by X-ray diffractometry (XRD) using a Panalytical X'pert Pro instrument, utilizing $\mathrm{Cu}-\mathrm{K} \alpha$ radiation $(\lambda=0.1542 \mathrm{~nm})$. To study the morphology, we performed plan-view scanning electron microscopy (SEM) measurement in aa Leo 1550 Gemini SEM instrument at an operating voltage ranging from $10 \mathrm{kV}$ to $20 \mathrm{kV}$ and a standard aperture value of $30 \mathrm{~mm}$. SEM images were analyzed using the ImageJ software [38] in order to quantify the substrate areal coverage and the size and shape of Ag features at the various nominal thicknesses.

The quality of epitaxial graphene before and after silver deposition, as well as the Ag thickness effect on the vibrational properties of epitaxial graphene, was evaluated by microRaman spectroscopy, including mapping analysis and statistical analysis. The Raman spectra were recorded by using micro-Raman setup based on a monochromator (Jobin-Yvon, model HR460) equipped with a CCD (couple-charged device) camera. The objective lens has a magnification of $100 \times$ and numerical aperture $\mathrm{NA}=0.95$ resulting in a $\sim 0.85 \mu \mathrm{m}$ diameter of the laser spot focused on the sample surface. A $532 \mathrm{~nm}$ diode-pumped solid-state laser with $1 \mathrm{~mW}$ power was used as an excitation source. The spectral resolution of the system is $\sim 5.5 \mathrm{~cm}^{-1}$. We observed the full disappearance of Raman signals from graphene underneath $30 \mathrm{~nm}$-thick silver film because thicker films become reflecting. In fact, this result indicates that continuous $\mathrm{Ag}$ films can be formed on graphene, but they are not of interest of this study because they mask 
the metal effect on graphene. Absorbance measurements were performed at room temperature using Shimatzu UV-2100 spectrometer.

The interaction of $\mathrm{Ag}$ species with epitaxial graphene on the $\mathrm{Si}$-face $4 \mathrm{H}-\mathrm{SiC}$ has been studied using hybrid density-functional theory calculations which were performed using the Gaussian 16 Rev. B.01 package [39]. We modelled epitaxial graphene by a cluster consisting of $4 \times 5$ graphene layer located above a $4 \times 5$ buffer layer on $4 \times 4$ Si-face surface of hexagonal SiC. According to this model, $26 \%$ of carbon atoms belonging to buffer layer is covalently bonded to $\mathrm{SiC}$ surface, forming $s p^{3}$ bonds, which is consistent with experimental observations [40, 41]. All the calculations were performed by using the PBE1PBE-D3 level of DFT [42, 43] with split basis set and empirical dispersion correction, which was included as Grimme's dispersion term [44]. The 6-31G basis set was used for carbon, silicon and hydrogen atoms, while the basis set developed by the LanL2DZ (Los Alamos National Laboratory 2 DoubleZeta) was used for $\mathrm{Ag}$ [45]. To shed more light on the nature of the interaction between $\mathrm{Ag}$ and epitaxial graphene, non-covalent interaction (NCI) and topological analyses were carried out using the Multiwfn software [46].

\section{Results and Discussion}

\section{A. Microstructure and morphology of Ag films on epitaxial graphene}

XRD patterns of $\mathrm{Ag}$ films of different nominal thicknesses grown on graphene/4H-SiC substrates are presented in Fig. 1. The results show that the Ag films are textured and they predominantly grow along the formed [111] crystallographic direction, as evidenced by the pronounced Ag (111) and (222) reflections. The intensity of these reflections increases with increasing film thickness, while weak (200) peaks appear for nominal thicknesses above $2 \mathrm{~nm}$. 


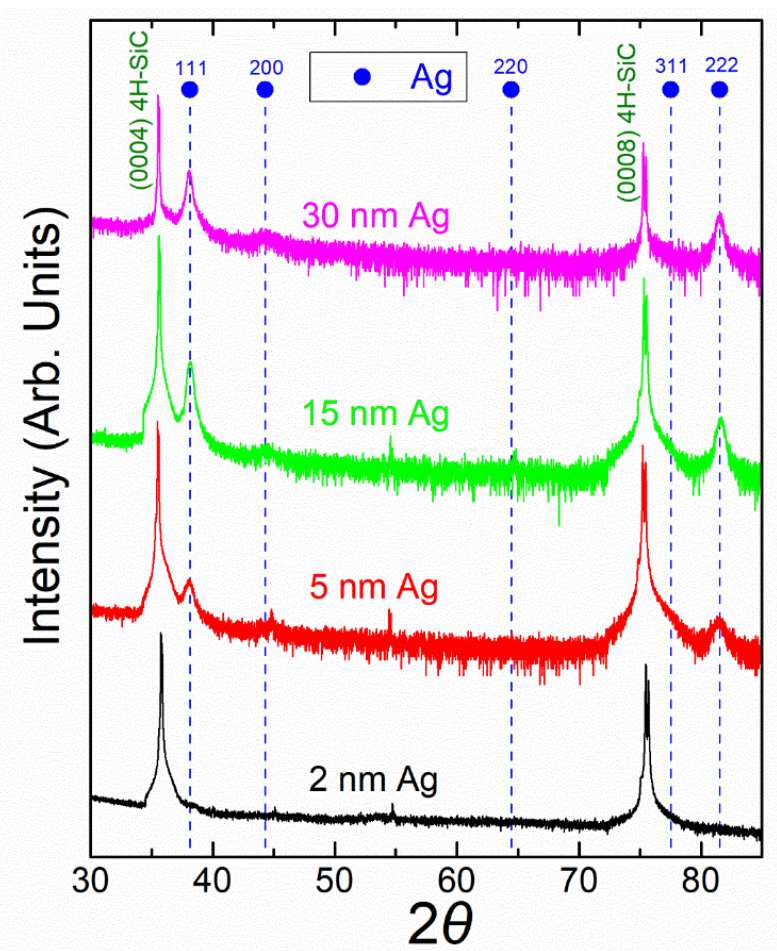

Figure 1. XRD patterns of Ag films with thickness ranging from $2 \mathrm{~nm}$ to $30 \mathrm{~nm}$, which were grown on epitaxial graphene at room temperature. The XRD peaks corresponding to FCC crystal structure of Ag are designated by filled blue circles.

The plan-view SEM images of Ag films with nominal thicknesses 2, 5, 10, 15, and $30 \mathrm{~nm}$ are show in Figs. 2 (a) through (e). The surface of the $2 \mathrm{~nm}$ film (Fig. 2(a)) consists of isolated circular islands. Analysis of multiple images corresponding to the same nominal thickness showed that the mean island size is $17.6 \mathrm{~nm}$ with standard deviation of 5.3. Increase of the nominal film thickness to $5 \mathrm{~nm}$ (Fig. 2(b)), yields elongated islands with mean size of $42.3 \mathrm{~nm}$ with standard deviation of 15.9. Further deposition of Ag results in increase of island size which form an interconnected network (10 and $15 \mathrm{~nm}$ in Figs. 2 (c) and (d), respectively) which eventually leads to the formation a continuous layer $(30 \mathrm{~nm}$; Fig. 2(e)). The morphological evolution observed in Fig. 2 is consistent with results for noble metal film growth on a variety of weakly-interacting substrates, including $\mathrm{SiO}_{2}$ and graphite [37, 47], and is result of the dynamic competition between island nucleation, growth, and coalescence [48]. 

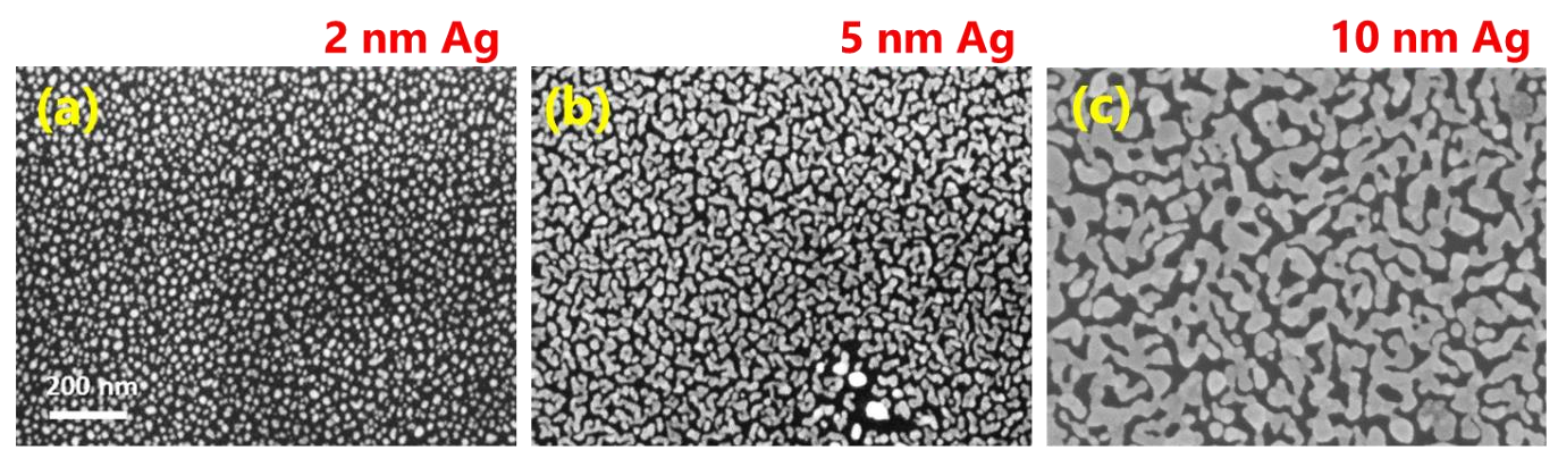

$15 \mathrm{~nm} \mathrm{Ag}$

$30 \mathrm{~nm} \mathrm{Ag}$
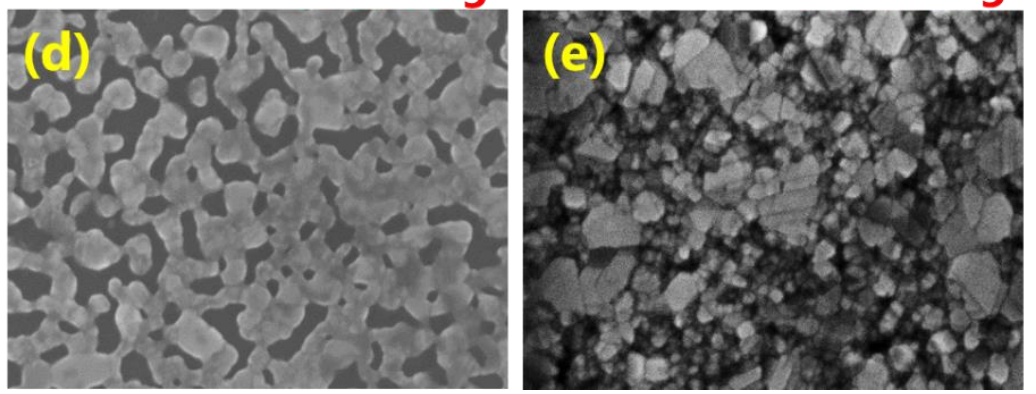

Figure 2. SEM images of the Ag films grown on epitaxial graphene corresponding to different nominal thicknesses: (a) $2 \mathrm{~nm}$, (b) $5 \mathrm{~nm}$, (c) $10 \mathrm{~nm}$, (d) $15 \mathrm{~nm}$ and (e) $30 \mathrm{~nm}$.

\section{B. Difference in Ag nucleation processes on bare $\mathrm{SiC}$ and epitaxial graphene:} experimental and theoretical insights

To shed more light on the role of epitaxial graphene for the Ag nucleation and growth, we compared film morphology on bare $4 \mathrm{H}-\mathrm{SiC}$ substrate vs. Morphology on graphene/4H-SiC. Analysis of the SEM images for $2 \mathrm{~nm} \mathrm{Ag}$ films on the two types of substrates showed that the mean diameter of $\mathrm{Ag}$ islands on bare $4 \mathrm{H}-\mathrm{SiC}$ is $11.3 \mathrm{~nm}$, with $\mathrm{RSD}=4.1$ (Fig. 3a), which is smaller than island size on epitaxial graphene (17.6 nm; Fig. 3b).

In order to understand the tendency of Ag islands toward larger sizes, we studied the adsorption behavior of four $\mathrm{Ag}$ atoms on both bare $\mathrm{SiC}$ and epitaxial graphene by means of density functional theory (DFT) calculations. Figures 3 (c) and (d) represent optimized structures of adsorbed atoms on 4H-SiC (Fig. 3(c)) and graphene/4H-SiC (Fig. 3(d)). It is seen that $\mathrm{Ag}$ species on bare $4 \mathrm{H}-\mathrm{SiC}$ tend to bind strongly to $\mathrm{Si}$ atoms, while the $\mathrm{Ag}$ species on epitaxial graphene form a tetramer metallic cluster in which metal-metal interaction prevails over the metal-graphene interaction. This means that epitaxial graphene template favors formation of Ag clusters, which can explain the larger island size observed experimentally and the smaller areal coverage for a given nominal thickness shown in Fig. 3(e). Furthermore, we notice the presence of the charge transfer from Ag cluster to epitaxial graphene (approximately 
$0.123 e^{-}$as was estimated by Hirshfeld population analysis), pointing out the $n$-type doping of graphene.

(a)
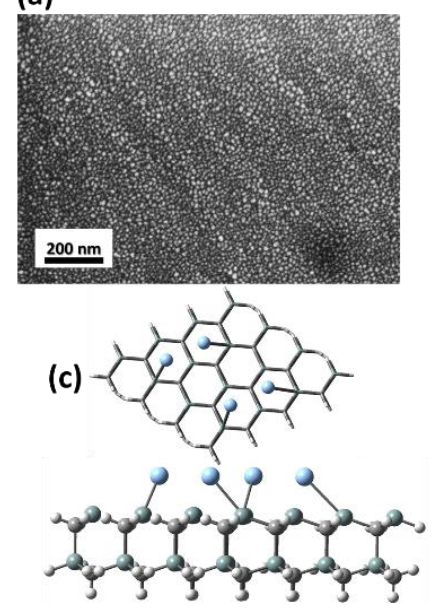

(b)
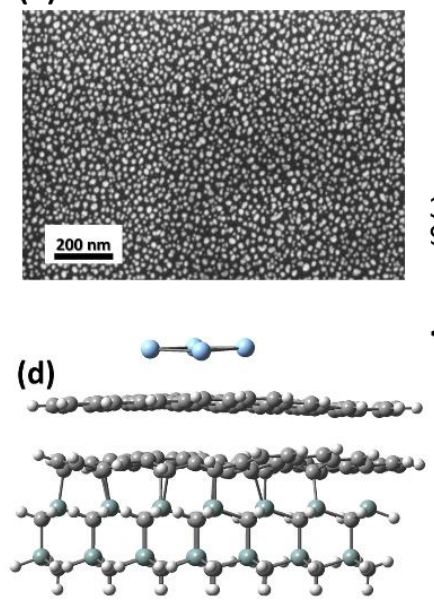

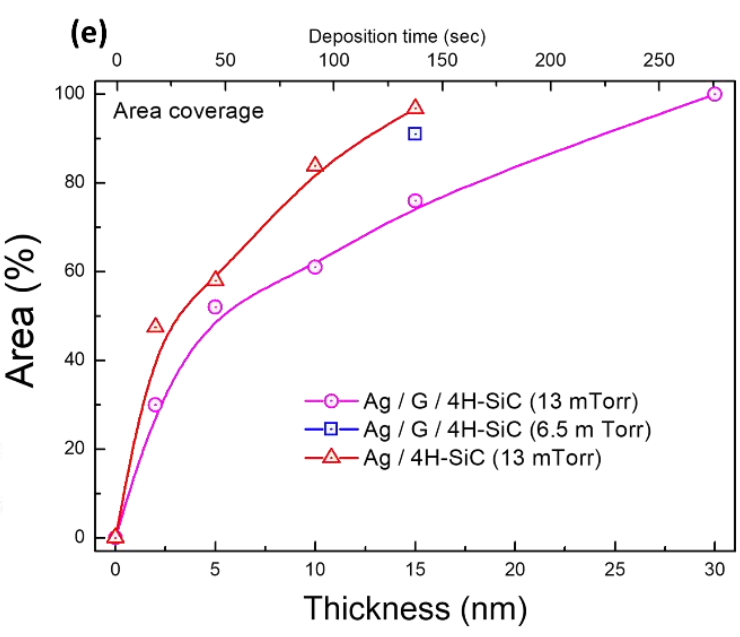

Figure 3. SEM images of the $2 \mathrm{~nm} \mathrm{Ag}$ films deposited on bare (a) $4 \mathrm{H}-\mathrm{SiC}$ and (b) graphene/4H$\mathrm{SiC}$ substrates. Optimized structures representing surface adsorption of the four $\mathrm{Ag}$ atoms on the bare (c) $4 \mathrm{H}-\mathrm{SiC}$ (d) graphene/4H-SiC surfaces. (e) Substrate areal coverage vs. nominal thickness on $4 \mathrm{H}-\mathrm{SiC}$ and graphene/4H-SiC substrates as extracted from SEM data.

To get deeper insight into the nature of interaction between $\mathrm{Ag}$ and both considered substrates, we performed non-covalent interaction (NCI) analysis (see the Figure S1 and related discussion in Figure S1) and revealed that the interaction between epitaxial graphene and $\mathrm{Ag}$ is dominated by weak long-range forces. In the absence of graphene on the surface of $4 \mathrm{H}-\mathrm{SiC}$, no maxima within this region are observed, which is indicative of chemisorption state rather than physisorption. The results are illustrated in Fig. S1. More evidence of the pronounced van der Waals interaction between $\mathrm{Ag}$ and epitaxial graphene can be obtained from topological analysis, which provides useful information on bond type (see Table 1 and related discussion in Supplementary Information). It was found that the interaction between Ag with epitaxial graphene is non-bonding in nature, while $\mathrm{Ag}-\mathrm{Si}$ demonstrates much stronger chemical bonding. In terms of experimental growth of Ag films, the above-mentioned results indicate that ideal graphene surface (free of defects and structural disorder) will provide a limited number of nucleation sites during sputtering process, thereby favoring lateral growth of Ag islands weakly bonded to substrate.

\section{Vibrational properties of epitaxial graphene before and after Ag deposition}

To better understand the nature of the interaction between Ag and epitaxial graphene, we consider the changes in the Raman spectra of epitaxial graphene induced by the deposition of 
Ag films. By performing Raman mapping analysis before and after Ag deposition, we constructed two-dimensional contour plots of the spectral data, showing the intensity of the Raman signal as a function of spatial coordinate (a lateral position of laser spot at the investigated region of the epitaxial graphene) and frequency (Figure 4). Such 2D plots are very useful to conclude about the homogeneity of Ag coverage. Contour plots of Raman data indicate that the intensity over 120 spectra is quite uniform (except some local regions displaying enhanced Raman signal from epitaxial graphene due to discontinuous nature of the Ag films), implying that the $\mathrm{Ag}$ islands are rather uniformly distributed over the investigated area of epitaxial graphene. The Raman spectra of the pristine epitaxial graphene are characterized by the presence of three most prominent spectral features: buffer-layer related features ranging from $1000 \mathrm{~cm}^{-1}$ to $1600 \mathrm{~cm}^{-1}, G$ peak (at $\left.1611 \mathrm{~cm}^{-1}\right)$ and $2 D$ peak $\left(2733 \mathrm{~cm}^{-1}\right)$. No Raman signal from the defect-related $D$ mode is observed before silver deposition, pointing to the high crystalline quality of the epitaxial graphene. This agrees well with the previous Raman studies of epitaxial monolayer graphene, which is originally $n$-type doped (typically $\sim 2-3 \times 10^{12} \mathrm{~cm}^{-2}$ and under biaxial compressive strain [49].

The Raman spectra of epitaxial graphene change in a continuous manner with increasing Ag-layer thickness. Particularly, a gradual redshift of both $G$ and $2 D$ peaks is observed, followed by subsequent spatial fluctuations in positions of characteristic lines. It is notable that the $2 \mathrm{D}$ line position is more sensitive to $\mathrm{Ag}$ deposition in comparison to the position of the characteristic $G$ peak. The peak amplitude ratio of $2 D$ to $G$ is found to decrease from 0.75 (for pristine graphene) to 0.1 and 0.06 for graphene decorated with $2 \mathrm{~nm}$ and $5 \mathrm{~nm}$ thick $\mathrm{Ag}$ film, respectively. This result and the previously mentioned red-shift of the $2 D$ peak indicate additional $n$-type doping of graphene [50], which is in line with literature data on Ag-induced $n$-doping in exfoliated graphene [51-53]. On the other hand, the variation in $2 D / G$ intensity ratio can be also interpreted as a result of the availability of plasmonic active noble metal nanoparticles at the graphene surface [54], which induces inequivalent enhancement of intensities of both modes (see related discussion below). Correlation analysis of the $G$ and $2 D$ Raman peaks allows to estimate the electron density concentration in epitaxial graphene [50, $55,56]$. The estimated electron density changes from $3.4 \times 10^{12} \mathrm{~cm}^{-2}$ (before Ag deposition) to $9.5 \times 10^{12} \mathrm{~cm}^{-2}$ and $1.3 \times 10^{13} \mathrm{~cm}^{-2}$ for graphene coverage with 2 and $5 \mathrm{~nm} \mathrm{Ag}$ layer. 

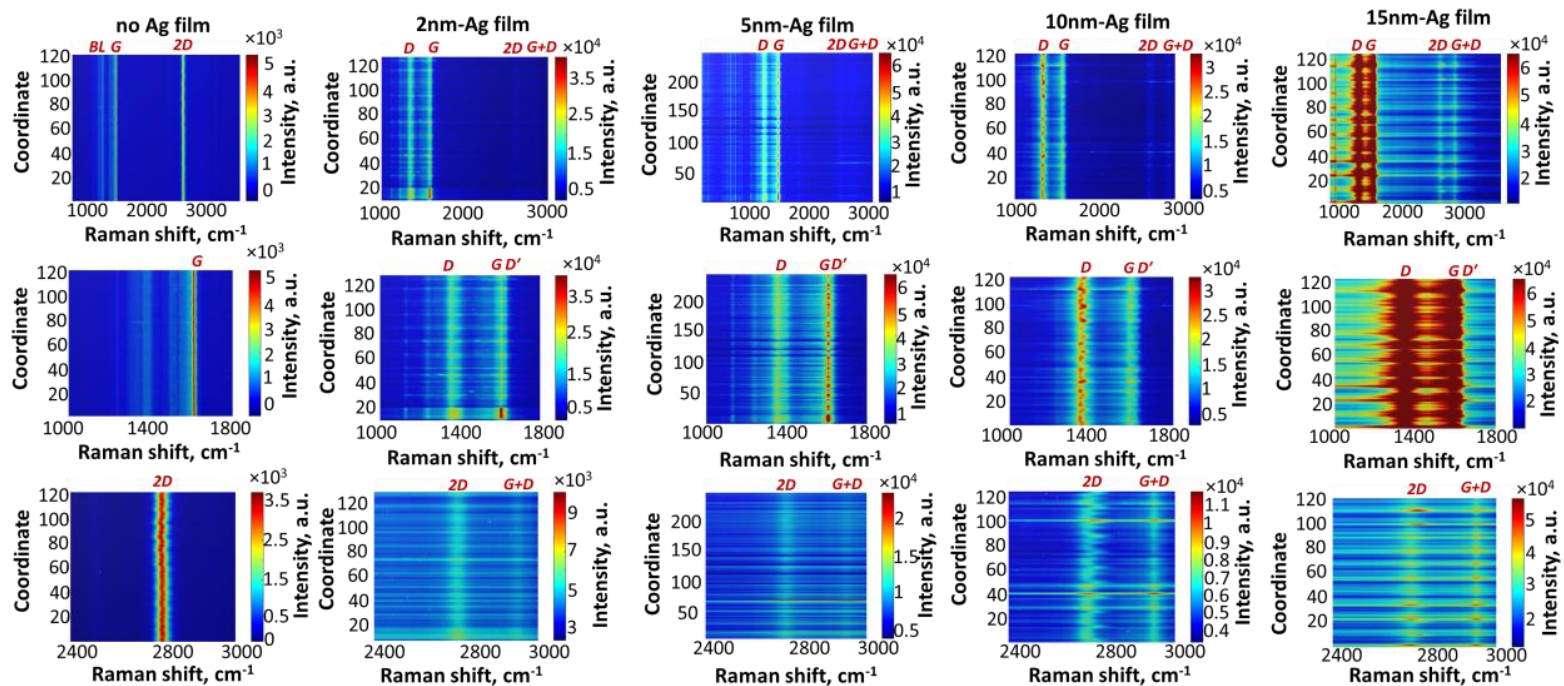

Figure 4. Two-dimensional contour plots of spectral data before and after Ag deposition, showing the intensity of Raman signal as a function of the Raman shift and coordinate. Raman fingerprint of the epitaxial graphene and the regions zooming near the $G$ and $2 D$ peaks are depicted in the three panels from the top to the bottom, respectively.

Deposition of the thicker films (nominal thicknesses $10 \mathrm{~nm}$ and $15 \mathrm{~nm}$ ), in which the islands are more interconnected and the substrate areal coverage is larger compared to the 2 and $5 \mathrm{~nm}$ films, results in higher values of $2 D / G$ ratio (0.29 and 0.4 , respectively) and reduced carrier density probably due to large mechanical strain at the interface. According to literature data on doping of free-standing graphene, the electron doping should result in a blue shift of the $G$ line and a decrease in its half-width. In our case, the red-shift of the $G$ line after $\mathrm{Ag}$ adsorption (Figure 5a-e) can be understood in terms of local strain model [57] and the broadening of the $G$ peak width can be related to inhomogeneous broadening due to the local strain variation caused by Ag-induced lattice distortions. According to the local strain model, the charge transfer between adsorbed Ag atoms and graphene, which induces the strain in graphene, is regarded as a main reason of the observed red-shift. Since the total charge transferred from $\mathrm{Ag}$ species to graphene surface increases with increasing the amount of $\mathrm{Ag}$ atoms (up to some level), local negatively charged epitaxial graphene regions occur near the reactive sites, while the positive charge will be accumulated at Ag species. Consequently, two different electrostatic forces are formed (Figure S2). One of them is attractive in nature and tends to bind the Ag atoms and/or clusters to the graphene surface via attraction between opposite charges, thereby causing a stretch of carbon-carbon bonds. According to our estimation $\mathrm{C}-\mathrm{C}$ bond lengths near the contact area in the case of single Ag atom adsorption are approximately $1.43 \AA$ that is $0.7 \%$ longer compared to that of perfect graphene. Another force 
is a repulsive in-plane force between the graphene regions with the same charges. Such negatively charged localized areas will be repelled by each other, thereby causing a strong tension in the graphene and occurrence of local strain that is mainly responsible for the redshift of the $G$ band.

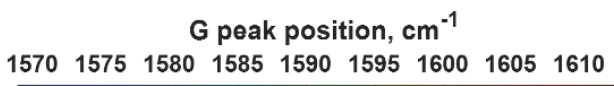

(a) no Ag on EG

$\begin{array}{ll}\text { (b) } 2 \mathrm{~nm} \mathrm{Ag} \text { on EG } & \text { (c) } 5 \mathrm{~nm} \text { Ag on EG }\end{array}$

$\begin{array}{lll}\text { (d) } 10 \mathrm{~nm} \mathrm{Ag} \text { on EG } & \text { (e) } 15 \mathrm{~nm} \mathrm{Ag} \text { on EG }\end{array}$
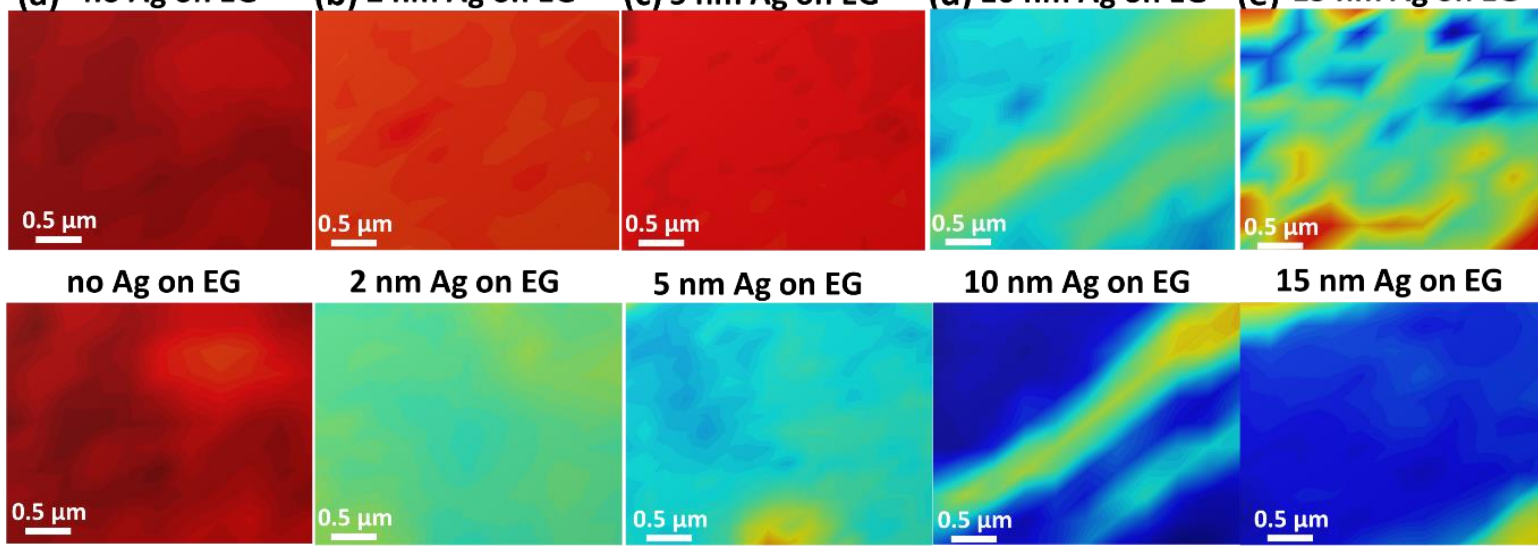

(f)

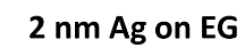

$5 \mathrm{~nm} \mathrm{Ag}$ on EG

$10 \mathrm{~nm} \mathrm{Ag}$ on EG

$15 \mathrm{~nm}$ Ag on EG

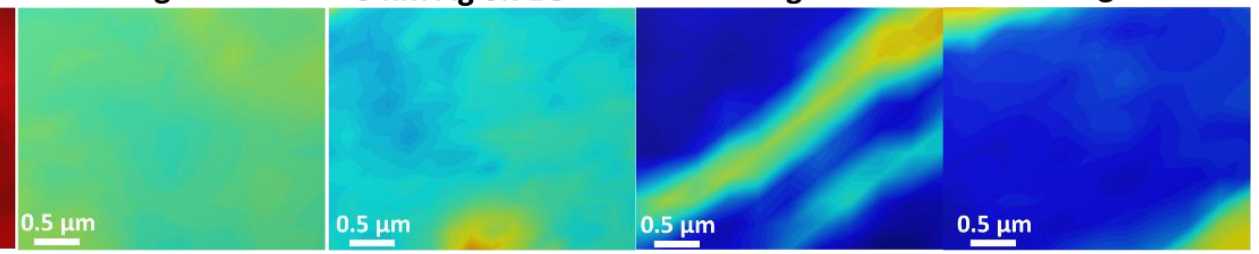

(g)

(h)

(i)

(j)

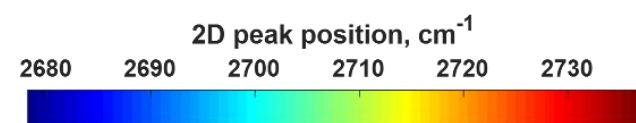

Figure 5. (Top panel) Raman spectral maps corresponding to the position of the $\mathrm{G}$ peak of epitaxial graphene before (a) and after Ag deposition: $2 \mathrm{~nm}$ (b), $5 \mathrm{~nm}$ (c), $10 \mathrm{~nm}$ (d) and $15 \mathrm{~nm}$ (e), respectively. (Bottom panel) Spectral position of the $2 D$ peak of epitaxial graphene before (f) and after Ag deposition: $2 \mathrm{~nm}(\mathrm{~g}), 5 \mathrm{~nm}(\mathrm{~h}), 10 \mathrm{~nm}$ (i) and $15 \mathrm{~nm}$ (j), respectively.

Another important observation is that all Ag-decorated epitaxial graphene samples exhibit defect-related Raman peaks: breathing $D$ mode, $D^{\prime}$ peak (weak low-frequency shoulder of the $G$ peak) and $D+G$ peak (see Figure 4). The increase in Ag film thickness gives rise to increase in the degree of disorder as manifest by the enhancement of intensity of the $D, D^{\prime}$, and $D+G$ peaks when moving from the left- to the right-hand-side panels in Fig. 4. The emergence of the disorder-related peaks is explained by defect-activated resonance Raman scattering by optical phonons near the $K$ point of the Brillouin zone - intervalley scattering from $K$ to $K^{\prime}(D$ peak), intravalley scattering from $K$ to $K\left(D^{\prime}\right.$ peak) and combination scattering ( $D+G$ peak) [58, 59] - and can be originating from the generation of point defects during magnetron sputtering process.

A detailed analysis of the relationships between defect-related and characteristic Raman peaks $(G$ and $2 D)$ can be used to determine the defect density and graphene domain size (the 
distance between two subsequent disruptions in graphene network, showing the geometrical size of the graphene region, where graphene remains intact) [60] as well as to elucidate the nature of defects [61]. In this context, the ratio of the intensities of the $D$ - and $G$-lines is indicative of the degree of structural perfection of the graphene film (Figure 6): the smaller this value is, the more perfect the film is. After plotting the experimental points $(D / G$ amplitude ratio values for Ag-decorated graphene), on the theoretical curve ( $D / G$ amplitude ratio versus domain size), experimentally derived domain size value can be easily extracted by projecting the intersections down to the $x$-axis (Figure 6e). Knowing the value of $L_{\mathrm{d}}$, one can estimate the defect density in epitaxial graphene [62]. In our case, the intensity ratio increases with increase in Ag-layer thickness (Figs. 6 (a)-(d)), suggesting reduction of domain size in graphene (Fig. 6e) and subsequent increase in density of defects. More specifically, for graphene decorated with $2 \mathrm{~nm}$ and $5 \mathrm{~nm} \mathrm{Ag}$ films the $D / G$ ratio is less than 1 and defect density is approximately equal to $3.0 \times 10^{11} \mathrm{~cm}^{-2}$ and $2.7 \times 10^{11} \mathrm{~cm}^{-2}$, respectively. Interfacing epitaxial graphene with thicker films $(10 \mathrm{~nm}$ and $15 \mathrm{~nm})$ leads to generation of larger number of defects $\left(7.7 \times 10^{11} \mathrm{~cm}^{-}\right.$ 2 and $9.5 \times 10^{11} \mathrm{~cm}^{-2}$, respectively).

To shed light on the nature of defects, we plotted the $D / G$ vs. $D^{\prime} / G$ amplitude ratios for all Ag-decorated epitaxial graphene samples (Fig. 6f). Curve fitting with linear regression indicates the slopes of the linear dependences belonging to graphene decorated with 2-10 nm Ag films is less than 2, which can be attributed to a formation of boundary-related defects, rather than to vacancy defects and/or $s p^{3}$ defects. We suggest that the so-called boundary-related defects can be referred to the case of substitutional doping of graphene, which modifies the inplane $\mathrm{C}-\mathrm{C}$ bonds. It is likely that during the initial film growth stages, Ag nucleation occurs preferentially at the domain boundaries, edges of the bilayer inclusions, and other linear native defects in epitaxial graphene, thereby avoiding the inert host sites of the basal plane of graphene. Since the atomic radius of $\mathrm{Ag}(160 \mathrm{pm})$ is much higher than the atomic radius of $\mathrm{C}(70 \mathrm{pm})$, whenever substitution doping occurs, it induces disorder in graphene and appearance of local tensile strains. Concomitantly, lateral growth of the initially deposited islands takes place and a part of the $\mathrm{Ag}$ atoms above defect-free region of graphene interacts with graphene mainly through dispersive forces, producing $n$-type doping without structural damages. As more and more Ag atoms arrive to epitaxial graphene surface, the islands grow, coalesce and form a continuous film. Under such conditions, Ag atoms are forced to occupy not only thermodynamically favorable sites of graphene, but also all available sites. As a result of such nucleation, the formation of on-site Ag-C bonds related to out-of-plane Ag atoms adsorbed onto graphene is highly probable. For this reason, after deposition of $15 \mathrm{~nm} \mathrm{Ag} \mathrm{films} \mathrm{epitaxial}$ 
graphene exhibits linear dependence of $D / G$ amplitude ratio versus $D^{\prime} / G$ amplitude ratio with a slope of $\sim 13$, which can be ascribed to $s p^{3}$ defects generation upon Ag adsorption followed by the formation of the continuous Ag film. To better understand the defects' energetics, we performed additional $a b$ initio calculations (see details in Supplementary Materials) using periodic boundary conditions (PBC). The optimized structures for defect-free and defectcontaining monolayer (ML) epitaxial graphene are presented in Figure S3. It is clearly seen that out-of-plane distortion caused by monovacancy formation in graphene is negligibly small, while the substitution of a carbon atom in the monolayer with Ag atom causes a significant distortion of the graphene plane followed by the protrusion of the Ag atom from the $s p^{2}$-bonded surface.

The formation energy of mentioned defects in epitaxial graphene is defined as:

$$
E_{f}=E_{d}-E_{p}+\sum_{i} n_{i} \mu_{i}
$$

where $E_{d}$ and $E_{p}$ are total energy of defective and defect-free perfect epitaxial graphene, respectively, $\mu_{i}$ is the chemical potential of the removed/added atom, which can be taken as the total energy of the isolated atom, and $n_{i}$ is the number of removed atoms to form monovacancy (positive value) or added adatoms to form $\mathrm{Ag}$ substitutional defect (negative value). The formation energy of single monovacancy in our calculations is estimated to be $\sim 17.7 \mathrm{eV}$, which is higher than previously reported values of 7.6-7.9 eV for monovacancy formation in freestanding infinite graphene [63]. On the other hand, the formation energy of Ag substitutional defects is found to be $13.3 \mathrm{eV}$, which explains their prevalence in 2-10 nm Ag film-covered graphene. Since the sputtered Ag target atoms in our experiment have energies in the order of few eV with energy tails of several tens of eV, their kinetic energies are lower than threshold energies for vacancy production, and $\mathrm{Ag}$ atoms tend to occupy the available in-plane nucleation sites. 


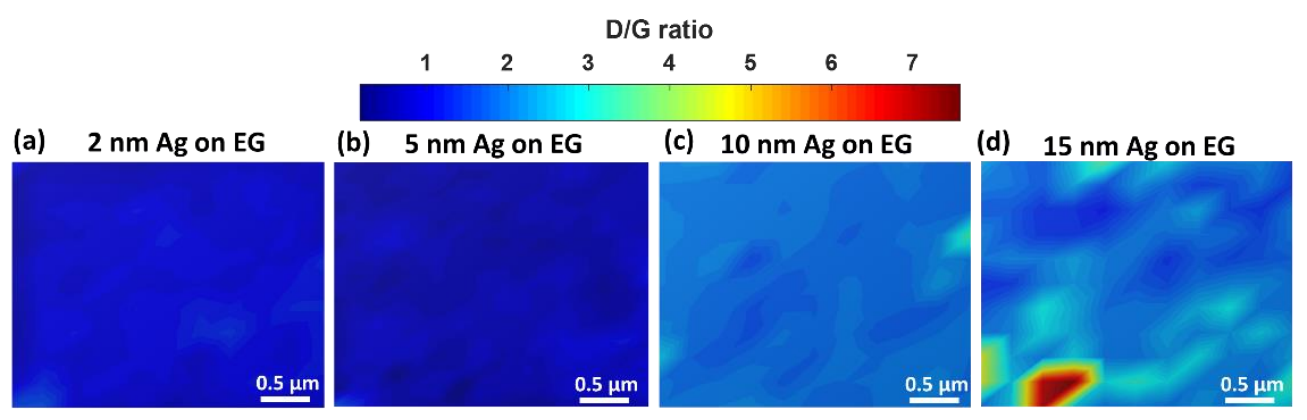

(e)
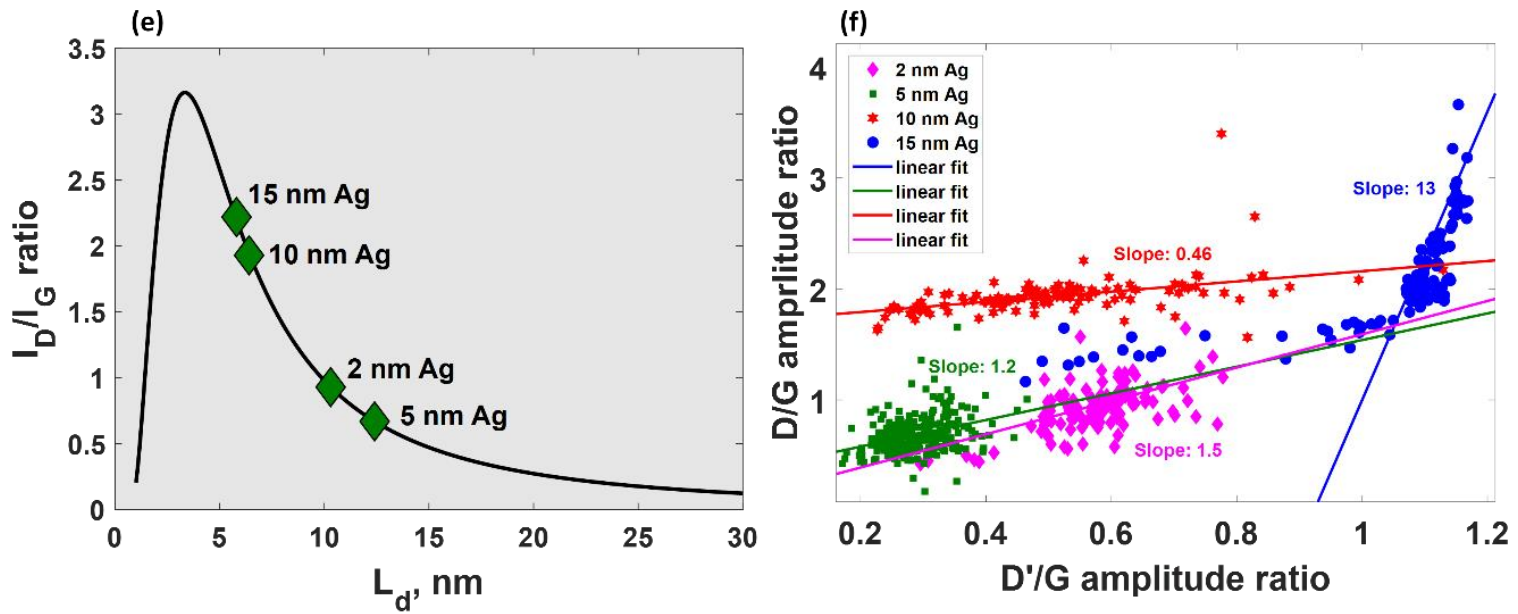

Figure 6. Intensity ratio maps of $D$ to $G$ band for epitaxial graphene after deposition of $\mathrm{Ag}$ films: (a) $2 \mathrm{~nm}$, (b) $5 \mathrm{~nm}$, (c) $10 \mathrm{~nm}$ and (d) $15 \mathrm{~nm}$. (e) Theoretical dependence of the $D / G$ amplitude ratio on the distance between two neighboring defects (graphene domain size) and experimental points corresponding to measured $D / G$ ratios for epitaxial graphene interfacing to silver thicknesses ranging from $2 \mathrm{~nm}$ to $15 \mathrm{~nm}$. (f) Mutual correlation between $D / G$ amplitude ratio on $D^{\prime} / G$ amplitude ratio for epitaxial graphene after deposition of $\mathrm{Ag}$ films with different thicknesses. The corresponding linear fits (solid lines) are also plotted.

By analyzing the dependencies of the $G$ and $2 D$ peak positions on $D / G$ amplitude ratio (Fig. 7), it is possible to trace the influence of defects on the characteristic vibrational modes. It is seen that $G$ peak is virtually insensitive to the presence of defects in graphene after decoration with $2 \mathrm{~nm}$ and $5 \mathrm{~nm} \mathrm{Ag}$ films (Fig. 7a), implying that the mentioned above red-shift of this peak can be attributed to a charge transfer between out-of-plane Ag dopants adsorbed onto epitaxial graphene rather than substitutional defects generated during early stages of $\mathrm{Ag}$ nucleation. Whilst $2 D$ peak for similar samples demonstrates more randomly distributed data points (Fig. 7b). After adsorption of thicker silver films, we observe a more complex picture, which is evidenced by large spread of data points and is contributed by both electron doping and local strains. 

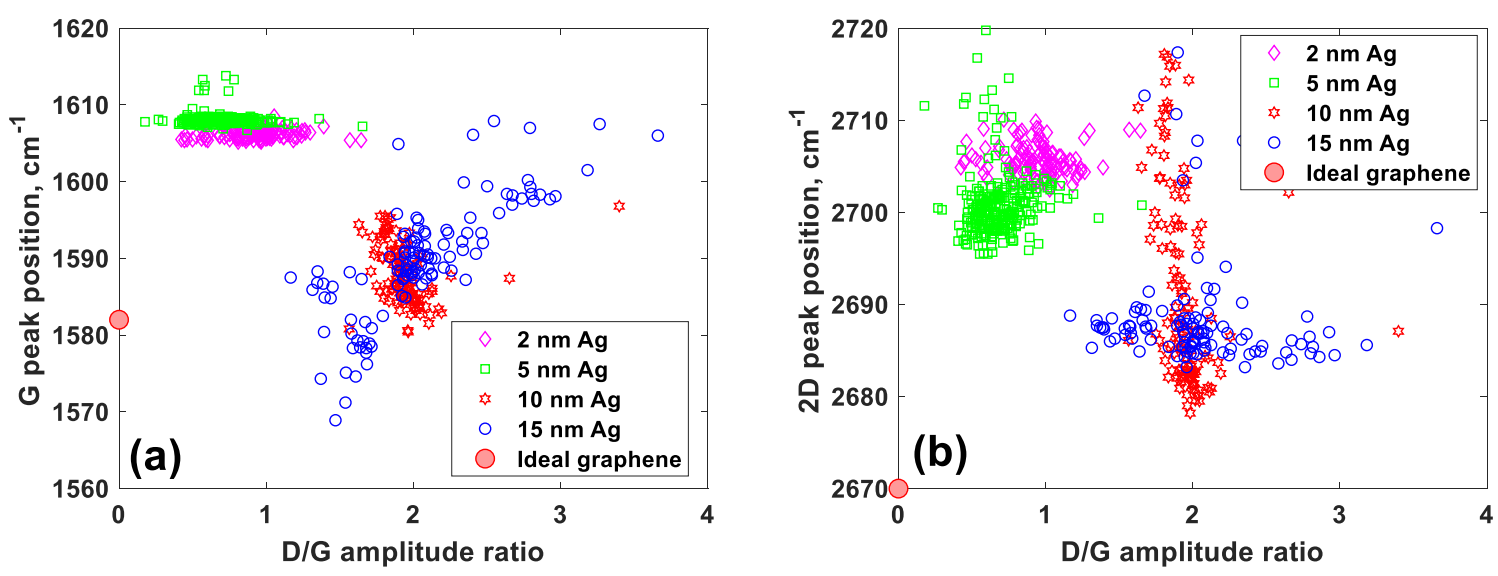

Figure 7. Dependences of (a) $G$ peak and (b) 2D peak positions on $D / G$ amplitude ratio for epitaxial graphene after deposition of Ag films with different thicknesses.

As a final note, it is striking that $G$ Raman mode displays an obvious enhancement in amplitude and peak area after deposition of Ag films at all nominal thicknesses (see Fig. 4), possibly due to SERS (Surface Enhanced Raman Scattering) effect [64]. G peak experiences the strongest enhancement because of the good matching between its position and the position of the $\mathrm{Ag}$ plasmon resonance peak. The degree of enhancement is not the same for the $G$ and $2 D$ modes, since the frequency of $2 D$ peak is away from the Ag resonance peak. However, the mechanism of SERS (observed in all Ag-decorated samples) is still questionable, since only morphology of $2 \mathrm{~nm}$ and $5 \mathrm{~nm} \mathrm{Ag}$ films (with mean particle size of $17.6 \mathrm{~nm}$ and $42.3 \mathrm{~nm}$, respectively) may, to some extent, provide the required conditions for SERS in terms of island size and interisland separation. At the same time, the morphology of the thicker Ag films (10 and $15 \mathrm{~nm})$ shows the presence of large interconnected islands and, thus, it would be hard to expect from these systems SERS phenomenon as a result of plasmonic effect. Absorbance measurements of Ag-covered graphene/4H-SiC (Figure S4, Supplementary Materials) confirmed the occurrence of LSPR, which is evidenced by enhanced absorbance when the 2-10 $\mathrm{nm} \mathrm{Ag}$ films were deposited onto epitaxial graphene/4H-SiC. Absorbance peak in the range 490 to $530 \mathrm{~nm}$ corresponds to the ranges, where LSPR for surface is reported. This, in combination with the morphology showing the existence of isolated particles at 2 and $5 \mathrm{~nm}$, speaks in favor of LSPR. SEM data show that at $10 \mathrm{~nm}$ the substrate surface features interconnected $\mathrm{Ag}$ islands, while inter-island distance decreases. This is consistent with the LSPR red-shift and broadening of the absorbance peak. Since the surface plasmon resonance is largely responsible for the Raman intensity enhancement [65], we consider the electromagnetic (EM) enhancement mechanism as 
dominant SERS mechanism for graphene/4H-SiC covered with small isolated nanoparticles (2 and $5 \mathrm{~nm}$ ), while Raman peak enhancement for samples coated with thicker Ag films can be explained by mixed contribution from charge-transfer resonance enhancement [66] and EM enhancement. According to literature data [67], the enhancement is expected to be maximal for a separated particle size of approximately $175 \mathrm{~nm}$, which is much larger than the mean radius of the islands in our films. We can foresee an eventual improvement across such a hybrid system, particularly through annealing of samples to reshape the silver nanoparticles [68].

\section{Conclusions}

Ag films of various thicknesses $(2-30 \mathrm{~nm})$ were prepared by magnetron sputtering technique on epitaxial graphene grown on (0001) 4H-SiC substrate and studied by XRD, SEM and Raman spectroscopy with the aim to gain understanding of graphene-metal interaction. Deposited films with a face-centered cubic crystal structure were formed by polycrystalline grains and their morphology was changed from nano-island like to interconnected structure as the thickness increases. $30 \mathrm{~nm} \mathrm{Ag}$ films showed to be optically reflective and were not subject to further studies. The comparison of early nucleation stages of $\mathrm{Ag}$ on bare $\mathrm{SiC}$ and epitaxial graphene as well as DFT calculations suggest that epitaxial graphene favors lateral growth of Ag due to weak van-der Waals interaction between them and strong metal-metal attraction. Very thin Ag films (2-5 nm) cause $n$-type doping of graphene evidenced by a systematic red shift of the $G$ and $2 D$ peak positions, while thicker films lead to increased strain. A model of local strain was proposed to explain unusual behavior of Raman modes, originating from the charge transfer from Ag to graphene. Ag deposition was found to induce a generation of in-plane defects (defects density of $\sim 3-7 \times 10^{11} \mathrm{~cm}^{-2}$ ) in epitaxial graphene on $\mathrm{SiC}$ decorated with 2-10 $\mathrm{nm} \mathrm{Ag}$ films and out-of-plane defects in epitaxial graphene decorated with $15 \mathrm{~nm} \mathrm{Ag}$ film (defect density of $\sim 9.5 \times 10^{11} \mathrm{~cm}^{-2}$ ). We interpreted the observation of the enhancement of $G$ peak amplitude and area for Ag-epitaxial graphene system as a synergetic SERS effect originating from both pronounced charge transfer at the interface and plasmonically-active $\mathrm{Ag}$ nanoparticles. The comparison of experimental findings and DFT calculations suggest that epitaxial graphene is a promising platform for the formation of novel $2 \mathrm{D}$ material systems with possible applications in electronics and sensorics.

\section{Acknowledgments}

The authors would like to thank financial support via VR grant 2018-04962, Project \# 302791 and SSF grants (SSF GMT14-0077, SSF RMA15-0024). IS acknowledges also the support from Ångpanneföreningens Forskningsstiftelse (Grant 16-541). KS acknowledges financial support from Linköping University (“LiU Career Contract, Dnr-LiU-2015-01510, 2015-2020”) 
and the Swedish research council (contract VR-2015-04630). KS and NP acknowledge financial support from the Olle Engkvist foundation (contract SOEB 190-312) and the WennerGren foundations (contract UPD2018-0071).

\section{References}

[1] N. Mounet, M. Gibertini, P. Schwaller, D. Campi, A. Merkys, A.Marrazzo, T.Sohier, I. E. Castelli, A.Cepellott, G. Pizzi and N. Marzari, Nat. Nanotechnol. 13 (2018) 246-252. https://doi.org/10.1038/s41565-017-0035-5.

[2] M. Faraji, M.Yousefi, S.Yousefzadeh, M. Zirak, N. Naseri, T.H. Jeon, W. Choi, A. Z. Moshfegh, Two-dimensional materials in semiconductor photoelectrocatalytic systems for water splitting, Energy Environ. Sci. 12 (2019) 59-95. https://doi.org/10.1039/C8EE00886H.

[3] K.S. Novoselov, D.V. Andreeva, W. Ren, G. Shan, Graphene and other two-dimensional materials, Front. Phys. 14 (2019) 13301. https://doi.org/10.1007/s11467-018-0835-6.

[4] J. Chen, E. Zhu, J. Liu, S. Zhang, Z. Lin, X. Duan, H. Heinz, Y. Huang, J. J. De Yoreo, Building two-dimensional materials one row at a time: Avoiding the nucleation barrier, Science 362, 6419 (2018) 1135-1139. https://doi.org/10.1126/science.aau4146.

[5] V.K. Sangwan, M.C. Hersam, Electronic Transport in Two-Dimensional Materials, Annu. Rev. Phys. Chem. 69 (2018) 299-325. https://doi.org/10.1146/annurev-physchem-050317021353.

[6] K.S. Novoselov, V.I. Fal'ko, L. Colombo, P. R. Gellert, M. G. Schwab \& K. Kim, A roadmap for graphene, Nature 490 (2012) 192-200. https://doi.org/10.1038/nature11458.

[7] C. R. Hammond, The Elements, in: D. R. Lide (Eds. ), Handbook of Chemistry and Physics (81st ed.). CRC press, Boca Raton, 2004.

[8] P. R. West, S. Ishii, G. V. Naik, Naresh K. Emani, V. M. Shalaev, A. Boltasseva, Searching for better plasmonic materials, Laser Photonics Rev. 4, 6 (2010) 795-808 https://doi.org/10.1002/lpor.200900055.

[9] H.J. Simon, D.E. Mitchell, J.G. Watson, Surface plasmons in silver films-a novel undergraduate experiment, Am. J. Phys. 43 (1975) 630. https://doi.org/10.1119/1.9764.

[10] Y. Jiang, S. Pillai, M.A. Green, Realistic Silver Optical Constants for Plasmonics. Sci. Rep. 6 (2016) 30605. https://doi.org/10.1038/srep30605.

[11] V.E. Calado, Z. Shou-En, S. Goswami, Q. Xu, K. Watanabe, T. Taniguchi, G.C.A.M. Janssen, L.M.K. Vandersypen, Ballistic transport in graphene grown by chemical vapor deposition. Appl. Phys. Lett. 104 (2014) 023103. https://doi.org/10.1063/1.4861627. 
[12] J. Baringhaus, M. Ruan, F. Edler, A. Tejeda, M. Sicot, A. Taleb-Ibrahimi, A.-P. Li, Z. Jiang, E. H. Conrad, C. Berger, C. Tegenkamp, W. A. de Heer, Exceptional ballistic transport in epitaxial graphene nanoribbons, Nature $506 \quad$ (2014) 349-354. https://doi.org/10.1038/nature12952.

[13] L. Pelaz, J.L. Orantes, J. Vicente, L. Bailón, J. Barbolla, Avalanche breakdown of highvoltage $p$ - $n$ junctions of SiC, Microelectron. J. 27 (1996) 43-51. https://doi.org/10.1016/00262692(95)00056-9.

[14] S. Chadhari, A. R. Graves, M. V. Cain, C. D. Stinespring, Graphene-based composite sensors for energy applications, Proc. SPIE 9836 (2016) 98360G-1+98360G-6. https://doi.org/10.1117/12.2223876.

[15] Y. Sekine, H. Hibino, K. Oguri, T.Akazaki, H.Kageshima, M. Nagase, K.-I. Sasaki, H.Yamaguchi, Surface-enhanced raman scattering of graphene on SiC, NTT Technical Review, 11 (2013) 1-6.

[16] X. Liu, Q. Fang, T. Hu, D. Ma, X. Zhang, S. Liu, F. Ma, K. Xu, Thickness dependent Raman spectra and interfacial interaction between $\mathrm{Ag}$ and epitaxial graphene on $6 \mathrm{H}-\mathrm{SiC}(0001)$, Phys. Chem. Chem. Phys., 20 (2018) 5964-5974. https://doi.org/10.1039/c7cp07338k.

[17] T. Suzuki, T. Itoh, S. Vantasin, S. Minami, Y. Kutsuma, K. Ashida, T.-A. Kaneko, Y. Morisawa, T. Miura, Y. Ozaki, Tip-enhanced Raman spectroscopic measurement of stress change in the local domain of epitaxial graphene on the carbon face of $4 \mathrm{H}-\mathrm{SiC}(000-1)$. Phys.Chem.Chem.Phys. 16 (2014) 20236. https://doi.org/10.1039/c4cp02078b.

[18] J. Nevalaita, P. KoskinenAtlas for the properties of elemental two-dimensional metals. Phys. Rev. B. 97 (2018) 035411. https://doi.org/10.1103/PhysRevB.97.035411.

[19] M. Amft, S. Lebègue, O. Eriksson, N.V. Skorodumova, Adsorption of Cu, Ag, and $\mathrm{Au}$ atoms on graphene including van der Waals interactions, J. Phys.: Condens. Matter. 23 (2011) 395001. https://doi.org/10.1088/0953-8984/23/39/395001.

[20] R.R. Ford, J. Pritchard, Work functions of gold and silver films. Surface potentials of mercury and xenon Trans, Faraday Soc. 67 (1971) 216-221. https://doi.org/10.1039/TF9716700216.

[21] P. Wang, D. Tanaka, S. Ryuzaki, S. Araki, K. Okamoto, K. Tamada, Silver nanoparticles with tunable work functions. Appl. Phys. Lett. $107 \quad$ (2015) 151601. https://doi.org/10.1063/1.4933253.

[22] I. Shtepliuk, T. Iakimov, V. Khranovskyy, J. Eriksson, F. Giannazzo, R. Yakimova, Role of the Potential Barrier in the Electrical Performance of the Graphene/SiC Interface, Crystals. 7 (2017) 162. https://doi.org/10.3390/cryst7060162. 
[23] S. Mammadov, J. Ristein, J. Krone, C. Raidel, M. Wanke, V. Wiesmann, F. Speck, T. Seyller, Work function of graphene multilayers on SiC(0001), 2D Mater. 4 (2017) 015043. https://doi.org/10.1088/2053-1583/4/1/015043.

[24] S.S. Sukumarana, C.R. Rekha, A.N. Resmi, K.B. Jinesh, K.G. Gopchandran, Raman and scanning tunneling spectroscopic investigations on graphene-silver nanocomposites, J. of Sci. Adv. Mater. Devices. 3 (2018) 353-358. https://doi.org/10.1016/j.jsamd.2018.06.003.

[25] S. Kopylov, A. Tzalenchuk, S. Kubatkin, V.I. Fal'ko, Charge transfer between epitaxial graphene and silicon carbide, Appl. Phys. Lett. $97 \quad$ (2010) 112109. https://doi.org/10.1063/1.3487782.

[26] X. Liu, T. Hu, Y. Miao, D. Ma, Z. Yang, F. Ma, K. Xu, P.K. Chu, Substitutional doping of $\mathrm{Ag}$ into epitaxial graphene on $6 \mathrm{H}-\mathrm{SiC}$ substrates during thermal decomposition, Carbon. 104 (2016) 233-240. https://doi.org/10.1016/j.carbon.2016.04.007.

[27] V. Amendola, Surface plasmon resonance of silver and gold nanoparticles in the proximity of graphene studied using the discrete dipole approximation method, Phys. Chem. Chem. Phys. 18 (2016) 2230-2241. https://doi.org/10.1039/c5cp06121k.

[28] H.-B. Sun, C. Fu, Y.-J. Xia, C.-W. Zhang, J.-H. Du, W.-C. Yang, P.-F. Guo, J.-Q. Xu, C.L. Wang, Y.-L. Jia, Enhanced Raman scattering of graphene by silver nanoparticles with different densities and locations, Mater. Res. Express. 4 (2017) 025012. https://doi.org/10.1088/2053-1591/aa59e5.

[29] X. Guo, L. Peng, L. Tang, J. Xiang, R. Ji, K. Zhang, C. M. Luk, S. K. Lai, R. Wan, Y. Duan, S. P. Lau, Large-area uniform electron doping of graphene by Ag nanofilm editors-pick, AIP Adv. 7 (2017) 045209. https://doi.org/10.1063/1.4979113.

[30] S. R. Syed, G.-H. Lim, S. J. Flanders, A.B. Taylor, B. Lim, J. W. M. Chon, Single layer graphene band hybridization with silver nanoplates: Interplay between doping and plasmonic enhancement, Appl. Phys. Lett. 109 (2016) 103103. https://doi.org/10.1063/1.4962401.

[31] P. Zhang, H. Wang, X. Zhang, W Xu, Y. Li, Qing Li, G. Wei, Z. Su, Graphene film doped with silver nanoparticles: self-assembly formation, structural characterizations, antibacterial ability, and biocompatibility, Biomater. Sci. 3 (2015) 852-860. https://doi.org/10.1039/c5bm00058k.

[32] N.F. Attia, A.M. Eid, M.A. Soliman, M. Nagy, Exfoliation and Decoration of Graphene Sheets with Silver Nanoparticles and Their Antibacterial Properties, J. Polyme. Environ. 26 (2018) 1072-1077. https://doi.org/10.1007/s10924-017-1014-5.

[33] M. Homa, N. Sobczak, J.J. Sobczak, A. Kudyba, G. Bruzda, R. Nowak, D. Giuranno, K. Pietrzak, M. Chmielewski, Interaction Between Liquid Silver and Graphene-Coated SiC 
Substrate, J. Mater. Eng. Perform. 27 (2018) 4140-4149. https://doi.org/10.1007/s11665-0183503-7.

[34] T. Hu, X. Liu, D. Ma, R. Wei, K. Xu, F. Ma, Formation of Micro- and Nano-Trenches on Epitaxial Graphene, Appl. Sci. 8 (2018) 2518. https://doi.org/10.3390/app8122518.

[35] R. Yakimova, T. Iakimov, M. Syväjärvi, Process for Growth of Graphene. U.S. Patent US9150417B2, 6 October 2015.

[36] I.G. Ivanov, J. Hassan, T. Iakimov, A.A. Zakharov, R. Yakimova, E. Janzén, Layernumber determination in graphene on $\mathrm{SiC}$ by reflectance mapping. Carbon. 77 (2014) 492-500. https://doi.org/10.1016/j.carbon.2014.05.054.

[37] V. Elofsson, B. Lü, D. Magnfält, E.P. Münger, and K. Sarakinos, Unravelling the physical mechanisms that determine microstructural evolution in ultra-thin Volmer-Weber films, J. Appl. Phys. 116 (2014) 044302. https://doi.org/10.1063/1.4890522

[38] M. D. Abràmoff, P. J. Magalhães, S. J. Ram, Image Processing with ImageJ, Biophotonics International, 11 (2004) 36-42.

[39] M. J. Frisch et al. Gaussian 16, Revision B.01, Wallingford, CT: Gaussian Inc, 2016.

[40] K.V. Emtsev, F. Speck, T. Seyller, L. Ley, J. D. Riley, Interaction, growth, and ordering of epitaxial graphene on $\mathrm{SiC}\{0001\}$ surfaces: a comparative photoelectron spectroscopy study, Phys. Rev. B. 77 (2008) 155303. https://doi.org/10.1103/PhysRevB.77.155303.

[41] C. Riedl, C. Coletti, U. Starke, Structural and electronic properties of epitaxial graphene on $\mathrm{SiC}(0001)$ : a review of growth, characterization, transfer doping and hydrogen intercalation, J. Phys. D: Appl. Phys. 43 (2010) 374009. https://doi.org/10.1088/0022-3727/43/37/374009.

[42] C. Adamo, V. Barone, Toward reliable density functional methods without adjustable parameters: the PBE0 model, J. Chem. Phys. $110 \quad$ (1999) 6158. https://doi.org/10.1063/1.478522.

[43] J.P. Perdew, K. Burke, M. Ernzerhof, Generalized gradient approximation made simple, Phys. Rev. Lett. 77 (1996) 3865. https://doi.org/10.1103/PhysRevLett.77.3865.

[44] S. Grimme, J. Antony, S. Ehrlich, H. Krieg, A consistent and accurate ab initio parametrization of density functional dispersion correction (DFT-D) for the 94 elements $\mathrm{H}-\mathrm{Pu}$, J. Chem. Phys. 132 (2010) 154104. https://doi.org/10.1063/1.3382344.

[45] P.J. Hay, W.R. Wadt, Ab initio effective core potentials for molecular calculations. Potentials for K to Au including the outermost core orbitale, J. Chem. Phys. 82 (1985) 299310. https://doi.org/10.1063/1.448975.

[46] T. Lu, F. Chen, Multiwfn: a multifunctional wavefunction analyzer, J. Comput. Chem. 33 (2012) 580-592. https://doi.org/10.1002/jcc.22885. 
[47] A. Jamnig, D.S. Sangiovanni, G. Abadias, and K. Sarakinos, Atomic-scale diffusion rates during growth of thin metal films on weakly-interacting substrates, Sci. Rep. 9 (2019) 6640. https://doi.org/10.1038/s41598-019-43107-8

[48] K. Sarakinos, A review on morphological evolution of thin metal films on weakly-

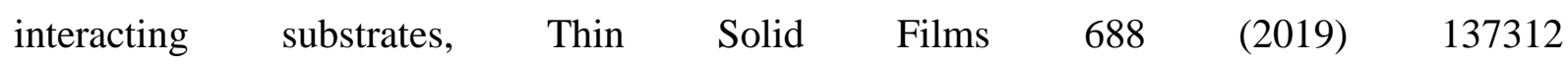
https://doi.org/10.1016/j.tsf.2019.05.031

[49] Z. H. Ni, W. Chen, X. F. Fan, J. L. Kuo, T. Yu, A. T. S. Wee, Z. X. Shen, Raman spectroscopy of epitaxial graphene on a SiC substrate, Phys. Rev. B. 77 (2008) 115416. https://doi.org/10.1103/PhysRevB.77.115416.

[50] A. Das, S. Pisana, B. Chakraborty, S. Piscanec, S.K. Saha, U.V. Waghmare, K.S. Novoselov, H.R. Krishnamurthy, A K. Geim, A.C. Ferrari \& A.K. Sood, Monitoring dopants by Raman scattering in an electrochemically top-gated graphene transistor, Nat. Nanotechnol. 3 (2008) 210-215. https://doi.org/10.1038/nnano.2008.67.

[51] A. Varykhalov, M. R. Scholz, T. K. Kim, O. Rader, Effect of noble-metal contacts on doping and band gap of graphene, Phys. Rev. B. 82 (2010) 121101(R). https://doi.org/10.1103/PhysRevB.82.121101.

[52] M. Papagno, P. Moras, P. M. Sheverdyaeva, J. Doppler, A. Garhofer, F. Mittendorfer, J. Redinger, C. Carbone, Hybridization of graphene and a Ag monolayer supported on $\operatorname{Re}(0001)$, Phys. Rev. B. 88 (2013) 235430. https://doi.org/10.1103/PhysRevB.88.235430.

[53] J. Lee, K.S. Novoselov, H.S., Shin, Interaction between Metal and Graphene: Dependence on the Layer Number of Graphene, ACS Nano. 5 (2011) 608-612. https://doi.org/10.1021/nn103004c.

[54] S. Sun, P. Wu, Competitive surface-enhanced Raman scattering effects in noble metal nanoparticle-decorated graphene sheets, Phys. Chem. Chem. Phys. 13 (2011) 21116-21120. https://doi.org/10.1039/C1CP22727K.

[55] D. M. Basko, S. Piscanec, and A. C. Ferrari, Electron-electron interactions and doping dependence of the two-phonon Raman intensity in graphene, Phys. Rev. B 80 (2009) 165413. https://doi.org/10.1103/PhysRevB.80.165413

[56] J. M. Urban, P. Dąbrowski, J. Binder, M. Kopciuszyński, A. Wysmołek, Z. Klusek, M. Jałochowski, W. Strupiński, and J. M. Baranowski, J. Appl. Phys. 115 (2014) 233504. https://doi.org/10.1063/1.4884015

[57] X. Zheng, W. Chen, G. Wang, Y. Yu, S. Qin, J. Fang, F. Wang, X.-A. Zhang, The Raman redshift of graphene impacted by gold nanoparticles, AIP Adv. 5 (2015) 057133. https://doi.org/10.1063/1.4921316. 
[58] R. Saito, M. Hofmann, G. Dresselhaus, A. Jorio, M.S. Dresselhaus, Raman spectroscopy of graphene and carbon nanotubes, Adv. Phys. 30 (2011) 413-550. https://doi.org/10.1080/00018732.2011.582251.

[59] R. Saito, A. Jorio, A. G. Souza Filho, G. Dresselhaus, M. S. Dresselhaus, M. A. Pimenta, Probing Phonon Dispersion Relations of Graphite by Double Resonance Raman Scattering, Phys. Rev. Lett. 88 (2002) 027401. https://doi.org/10.1103/PhysRevLett.88.027401.

[60] L.G. Cançado, K. Takai, T. Enoki, M. Endo, Y.A. Kim, H. Mizusaki, A. Jorio, L.N. Coelho, R. Magalhães-Paniago, M.A. Pimenta, General equation for the determination of the crystallite size La of nanographite by Raman spectroscopy, Appl. Phys. Lett. 88 (2006) 163106. https://doi.org/10.1063/1.2196057.

[61] A. Eckmann, A. Felten, A. Mishchenko, L. Britnell, R. Krupke, K.S. Novoselov, C. Casiraghi, Probing the Nature of Defects in Graphene by Raman Spectroscopy, Nano Lett. 12 (2012) 3925-3930. https://doi.org/10.1021/nl300901a.

[62] M. Yu.Vagin, A. N.Sekretaryova, I. G.Ivanov, A.Håkansson, T.Iakimov, M. Syväjärvi, R.Yakimova, I. Lundström, M. Eriksson, Monitoring of epitaxial graphene anodization, Electrochimica Acta. 238 (2017) 91-98. https://doi.org/10.1016/j.electacta.2017.04.016

[63] S. T. Skowron, I. V. Lebedeva, A. M. Popov, and E. Bichoutskaia, Energetics of atomic scale structure changes in graphene, Chem. Soc. Rev., 2015, 44, 3143-3176. https://doi.org/10.1039/C4CS00499J

[64] F. Schedin, E. Lidorikis, A. Lombardo, V.G. Kravets, A.K. Geim, A.N. Grigorenko, K.S. Novoselov, Andrea C. Ferrari, Surface-Enhanced Raman Spectroscopy of Graphene. ACS Nano. 4 (2010) 5617-5626. https://doi.org/10.1021/nn1010842.

[65] A. N. Sidorov, G. W. Sławiński, A.H. Jayatissa, F. P. Zamborini, G. U.Sumanasekera, A surface-enhanced Raman spectroscopy study of thin graphene sheets functionalized with gold and silver nanostructures by seed-mediated growth, Carbon 50 (2012) 699 . https://doi.org/10.1016/j.carbon.2011.09.030

[66] S. Sun, P. Wu, Competitive surface-enhanced Raman scattering effects in noble metal nanoparticle-decorated graphene sheets, Phys. Chem. Chem. Phys. 13 (2011) 21116-21120. https://doi.org/10.1039/C1CP22727K.

[67] A. Urich, A. Pospischil, M.M. Furchi, D. Dietze, K. Unterrainer, T. Mueller, Silver nanoisland enhanced Raman interaction in graphene, Appl. Phys. Lett. 101 (2012) 153113. https://doi.org/10.1063/1.4758696. 
[68] S.J. Park, S.J. Kang, Surface-plasmon Resonance-wavelength Control of graphene Surfaces Containing Metal Nanoparticles, Journal of the Korean Physical Society. 63 (2013) 59. https://doi.org/10.3938/jkps.63.5. 